was put upon the colon, another upon the ileum, and the intermediate portion of intestine removed. Upon examination it was found that the lower portion of the ascending colon, where it is continuous with the caput coli, was turned inwards upon itself, and that this inflected portion inclosed the coecum, which was again in part everted upon itself, or turned (so to speak) "inside out ;" the appendix vermiformis and about an inch and a half of the termination of the ileum, were included in the colon. The coats of the coecum were exceedingly congested; the mucous membrane very soft and swollen; the thickness of the gut much increased, and the cellular tissue between its coats infiltrated with serum. There was within the colon a small quantity of thin brownish-yel. low fluid.

Dr. Chowne mentioned this case, not as very rare, but as presenting peculiarities which are more characteristic of intussusceptio than of obstruction within the bowels from any other cause, although it could not be considered as a common occurlence that a lump should be felt in the abdomen; and although absence of such lump would not negative the supposition that intus-susception existed, yet the presence of a hard substance very much strengthened the suspicion, and a gradual but pretty constant escape of bloody fluid from the rectum constituted a strong indication of that condition.

After a short address from the president, Mr. H.J. Johnson, the society adjourned until October next.

\section{PROFESSOR GROVES'S NEW}

$$
\text { BATTERY, }
$$

IN REFERENCE TO THE

\section{ELECTRICITY OF A N M ALS.}

To the Editor of THE Lancet.

SIR,-On reading over Professor Groves's experiments with his new gaseous voltaic battery, a point has struck me, with regard to the source of the electricity in animal bodies,-which no doubt exists whether it be identical with the nervous influence or not. In the voltaic pile it is certainly the oxygenation of a metal that greatly increases, if not actually causes, the evolution of the electric influence; but the oxygenation of the blood is a chemical action, so entirely different from this, that it always seemed very doubtful whether we could logically infer that the combination of oxygen in this case likewise produced electricity. Accordingly, Dr. Prout, many years ago, only, $\mathrm{I}$ believe, broached the hypothesis as a probability. Now, Professor Groves's discovery seems to have brought us one step nearer certainty on this point, for it shows that when oxygen

* Phil, Magazine, Dec. 1842. combines with a gas, and forms water, electricity is likewise produced ; for Mr. Groves has produced the shock, spark, chemical decompositions, \&c., by fifty pairs of the plati. num oxygen-hydrogen battery - has decomposed the iodide of potassium, indeed, by four pairs only. Organic chemists, therefore, have only to show that water is actually formed by the union of the oxygen absorbed with hydrogen in any part of the circulating system, and they have at once proved that a good deal of electricity must be disengaged. But further, this discovery of Mr. Groves's has enabled us also to affirm, as certainly as can be done without experiment, that electricity is also evolved when oxygen, by uniting with carbonic oxide, forms carbonic acid; for platinum is well known also to have the power of causing the formation of carbonic acid as well as water, when carbonic oxide is let up with a mixture of oxygen and hydrogen; indeed, to form this before it forms uater. (Dr. Henry, Phil. Trans., 1824.) Now, although Mr. Groves does not appear to have tried carbonic oxide in his battery, there can be little doubt (as platinum makes this combine with oxygen before it makes hydrogen) that much electricity is evolved by the formation of carbonic acid at low temperatures; for, I believe, many years ago Laplace showed that it was by the combustion of carbon. It is also remarkable, and in favour of this general view of the production of electricity by respiration, that oletiant gas is also acted upon, though not until after the hydrogen and oxygen have united, and carburetted hydrogen not at all. (Henry, Phil. Trans.) Now, it is far more probable that carbonic oxide is formed in the course of the circulation than either of the above gases.

It may be objected to the abova that we have no platinum in the animal system; but the platinum, properly considered, seems only to be regarded as, in some way or another (for Mr. Groves asks, Would not gold and silver act?), as a conductor, making the electricity evolved evident. "The gases diminish, and the hydrogen much more rapidly than the oxygen;" so here is the oxidation of the hydrogen, and this only. I now have only to add that, nevertheless, $M$. Schoenbein "thinks (Phil. Mag., March, 1843 ,) that oxygen does not contribute to the current." He admits, however, that he has not yet tried the experiment of having all the tubes filled with hydrogen; but says that hydrogen and water produce a current when platinum completes the circuit. However, Mr. Groves tried common air in all the tubes, carbonic acid and nitrogen, and oxygen and nitrogen in alternate tubes, with no effect, and his theory seems to be that the union of oxygen and hydrogen is the cause of the current. I am, Sir, your most obedient servant,

May, 1843.
H, Prater. 